\title{
Inflammatory and Metabolic Dysregulation and the 2-Year Course of Depressive Disorders in Antidepressant Users
}

\author{
Nicole Vogelzangs*, ${ }^{*, 2}$, Aartjan TF Beekman', Arianne KB van Reedt Dortland', Robert A Schoevers ${ }^{3}$, \\ Erik J Giltay ${ }^{4}$, Peter de Jonge ${ }^{3}$ and Brenda WJH Penninx ${ }^{1,2}$ \\ 'Department of Psychiatry and EMGO Institute for Health and Care Research, VU University Medical Center, Amsterdam, \\ The Netherlands; ${ }^{2}$ Neuroscience Campus Amsterdam, VU University Medical Center, Amsterdam, The Netherlands; ${ }^{3}$ Department of Psychiatry, \\ University Medical Center Groningen, University of Groningen, Groningen, The Netherlands; ${ }^{4}$ Department of Psychiatry, Leiden University Medical \\ Center, Leiden, The Netherlands
}

\begin{abstract}
Scarce evidence suggests that inflammatory and metabolic dysregulation predicts poor response to antidepressants, which could result in worse depression outcome. This study prospectively examined whether inflammatory and metabolic dysregulation predicted the 2-year course of depressive disorders among antidepressant users. Data were from the Netherlands Study of Depression and Anxiety, including 315 persons ( $18-65$ years) with a current depressive disorder (major depressive disorder, dysthymia) at baseline according to the DSMIV criteria and using antidepressants. Inflammatory (C-reactive protein, interleukin-6 (IL-6), tumor-necrosis factor- $\alpha$ ) and metabolic (waist circumference, triglycerides, high-density lipoprotein (HDL) cholesterol, blood pressure, fasting glucose) factors were measured at baseline. Primary outcome for course of depression was indicated by whether or not a DSM-IV depressive disorder diagnosis was still/ again present at 2-year follow-up, indicating chronicity of depression. Elevated IL-6, low HDL cholesterol, hypertriglyceridemia, and hyperglycemia were associated with chronicity of depression in antidepressant users. Persons showing $\geqslant 4$ inflammatory or metabolic dysregulations had a 1.90 increased odds of depression chronicity $(95 \% \mathrm{Cl}=1.12-3.23)$. Among persons who recently (ie, at most 3 months) started antidepressant medication $(N=103)$, having $\geqslant 4$ dysregulations was associated with a 6.85 increased odds of depression chronicity $(95 \% \mathrm{Cl}=1.95-24.06)$. In conclusion, inflammatory and metabolic dysregulations were found to predict a more chronic course of depressive disorders among patients using antidepressants. This could suggest that inflammatory and metabolic dysregulation worsens depression course owing to reduced antidepressant treatment response and that alternative intervention treatments may be needed for depressed persons with inflammatory and metabolic dysregulation.

Neuropsychopharmacology (2014) 39, 1624-1634; doi: I0.1038/npp.20 I4.9; published online 12 February 2014
\end{abstract}

Keywords: inflammation; metabolic syndrome; depression; antidepressants; prospective cohort

\section{INTRODUCTION}

Depressive disorders are among the most prevalent psychiatric disorders (Kessler et al, 2012) and rank high in the top 10 of diseases with the largest disease burden worldwide (Mathers and Loncar, 2006). Depression can have huge effects on well-being and daily functioning of the individual (Buist-Bouwman et al, 2006) and often presents with a chronic-intermittent course (Rhebergen et al, 2009; Spijker et al, 2002). Over a 2-year time period, more than half of depressed persons show recurrence or persistence of depression, despite treatment efforts (Penninx et al, 2011). Severity, duration, comorbidity, and an early onset have been shown to predict an unfavorable course (Penninx et al,

*Correspondence: Dr N Vogelzangs, Department of Psychiatry and EMGO Institute for Health and Care Research, VU University Medical Center, AJ Ernststraat I 187, I08 I HL Amsterdam, The Netherlands, Tel: + 3120788 452I, Fax: + 31207885664 ,

E-mail: n.vogelzangs@vumc.nl/n.vogelzangs@ggzingeest.nl

Received 19 July 2013; revised 27 November 2013; accepted 16 December 2013; accepted article preview online 20 January 2014
2011). Although these factors can help identify those patients at risk for poor outcome, they do not contribute to a better understanding of the pathophysiology of depression chronicity. Insight into pathophysiological processes may facilitate the development of new interventions to improve the course of this disabling disorder, in particular for those not responding to current treatments.

Recently, attention has focused on inflammatory and metabolic dysregulation in depression. As inflammatory and metabolic processes are highly linked (Sutherland et al, 2004 ), it is of great value to consider them together. Metaanalyses have shown increased inflammatory and metabolic factors in depressed persons (Dowlati et al, 2010; Howren et al, 2009; Pan et al, 2012). Most of the existing research is based on cross-sectional data, making it impossible to unravel the direction of potentially causal associations. Sparse evidence suggests that inflammatory and metabolic dysregulation can be found in particular among those with more chronic forms of depression (Duivis et al, 2011; Viinamaki et al, 2009; Vogelzangs et al, 2010, 2011). Limited evidence also exists that inflammation may be associated 
with a poor response to antidepressants, which in turn may contribute to a more chronic depressive disorder course. One small study in 24 major depressive disorder in-patients showed that higher interleukin (IL)-6 blood levels predicted non-response to a 6-week treatment with amitriptyline, whereas tumor necrosis factor (TNF)- $\alpha$ blood levels were high in both responders and non-responders, but only decreased during treatment in responders (Lanquillon et al, 2000). In another study among 100 major depressive disorder patients, higher serum levels of TNF- $\alpha$ predicted non-response to a 12-week treatment with escitalopram (Eller et al, 2008). Furthermore, a recent study among 74 depressed patients found that expression of genes related to inflammation predicted non-response to a 8-week treatment with escitalopram or nortriptyline (Cattaneo et al, 2013). Lastly, the IL- $1 \beta$ gene ( $C-511 T$, rs19644 and rs1143643) has been shown to predict antidepressant non-response (Baune et al, 2010; Yu et al, 2003).

The present study aimed to investigate whether inflammatory and metabolic dysregulation predicted a chronic course of depression among antidepressant users, which would be indicative of non-response to antidepressants. Using a large cohort of persons with current depressive disorders on antidepressant medication, we prospectively examined whether inflammatory (C-reactive protein (CRP), IL-6, TNF- $\alpha$ ) and metabolic (abdominal obesity, dyslipidemia, hypertension, hyperglycemia) dysregulation predicted the recurrence or persistence of a depressive disorder over 2 years.

\section{MATERIALS AND METHODS}

\section{Sample}

The Netherlands Study of Depression and Anxiety (NESDA) is a multisite naturalistic cohort study that aims to examine the long-term course of depressive and anxiety disorders and includes 2981 persons aged 18-65 years at the baseline assessment in 2004-2007. Persons from different developmental stages of psychopathology as well as controls participated, recruited from the community (19\%), general practice $(54 \%)$, and secondary mental health care $(27 \%)$. Persons with insufficient command of the Dutch language or a primary clinical diagnosis of bipolar disorder, obsessive compulsive disorder, severe substance use disorder, psychotic disorder, or organic psychiatric disorder, as reported by themselves or their mental health practitioner, were excluded. A detailed description of the NESDA study design and sampling procedures can be found elsewhere (Penninx et al, 2008). The research protocol was approved by the Ethical Committee of participating universities, and after complete description of the study, all respondents provided written informed consent. After 2 years, a face-to-face follow-up assessment was conducted with a response of $87.1 \%(N=2596)$. Non-response was higher in those with younger age, lower education, nonNorth European ancestry, and depressive disorder, but not associated with sex or anxiety disorder (Lamers et al, 2012).

For the present study, we selected persons who at baseline had a current depressive disorder diagnosis and were using antidepressants. Depressive disorder diagnosis (major depressive disorder, dysthymia) was established using the
Composite Interview Diagnostic Instrument (CIDI) according to the DSM-IV criteria (American Psychiatric Association, 2001). The CIDI is a highly reliable and valid instrument for assessing depressive disorders (Wittchen, 1994) and was administered by specially trained research staff. In addition, using the Life Chart Interview (LCI) (Lyketsos et al, 1994), the presence of depressive symptoms during the year before baseline was determined. A current disorder was defined as a 6-month CIDI depressive disorder diagnosis at baseline while confirming symptoms in the month before baseline at either the CIDI recency questions or the LCI. Medication use in the month before baseline was registered by inspection of drug containers brought in and coded according to the World Health Organization Anatomical Therapeutic Chemical (ATC) classification system (WHO Collaborating Centre for Drug Statistics Methodology, 2013). Antidepressant medication included use of selective serotonin reuptake inhibitors (SSRIs; $\mathrm{N} 06 \mathrm{AB})$, serotonin-norepinephrine reuptake inhibitors (SNRIs; N06AX16, N06AX21), tricyclic antidepressants (TCAs; N06AA), and tetracyclic antidepressants (TeCAs; N06AX03, N06AX05, N06AX11). Of the 890 persons with a current depressive disorder, 387 were using antidepressants, of whom 315 participated in the follow-up interview $(81.4 \%)$. All 315 had data available of at least one inflammatory or metabolic factor, and therefore constitute the sample for the present study.

\section{Inflammatory and Metabolic Factors}

Inflammatory markers were assessed at baseline and included CRP, IL-6, and TNF- $\alpha$. Blood samples were obtained in the morning after an overnight fast between 0800 and 0900 hours (mean 0845 hours $(S D=0: 15)$ using EDTA tubes and kept frozen at $-80{ }^{\circ} \mathrm{C}$. CRP and IL- 6 were assayed at the Department of Clinical Chemistry, VUmc. High-sensitivity plasma CRP levels were measured in duplicate by an in-house ELISA based on purified protein and polyclonal anti-CRP antibodies (Dako, Glostrup, Denmark). Plasma IL-6 levels were measured in duplicate by a high-sensitivity ELISA (PeliKine Compact ELISA; Sanquin, Amsterdam, The Netherlands). Plasma TNF- $\alpha$ levels were assayed in duplicate at Good Biomarker Science (Leiden, The Netherlands), using a high-sensitivity solidphase ELISA (Quantikine HS Human TNF- $\alpha$ Immunoassay; R\&D Systems, Minneapolis, MN). Intra- and interassay coefficients of variation were 5 and $10 \%$ for CRP, 8 and $12 \%$ for IL- 6 , and 10 and $15 \%$ for TNF- $\alpha$. Continuous measures of CRP, IL- 6 , and TNF- $\alpha$ were ln-transformed to obtain normal distributions. Additional dichotomous measures were constructed indicating high inflammation by using the median as cutoff (CRP $\geqslant 1.58 \mathrm{mg} / \mathrm{l}$; IL- $6 \geqslant 0.95 \mathrm{pg} / \mathrm{ml}$; TNF- $\alpha \geqslant 0.80 \mathrm{pg} / \mathrm{ml}$ ).

Metabolic factors were also measured at baseline. Waist circumference was assessed using a measuring tape to the nearest $0.1 \mathrm{~cm}$ midway between the lower rib margin and the iliac crest following normal expiration, upon light clothing. Triglyceride, high-density lipoprotein (HDL) cholesterol, and glucose levels were measured in morning fasting blood samples using routine standardized laboratorial methods. Blood pressure was averaged over two readings measured in a supine position. In accordance with 
the updated National Cholesterol Education Program-Adult Treatment Panel III guidelines (Grundy et al, 2005), five dichotomous metabolic dysregulations were defined: abdominal obesity (waist circumference $>88 / 102 \mathrm{~cm}$ (women/ men)); hypertriglyceridemia (triglycerides $\geqslant 1.70 \mathrm{mmol} / \mathrm{l}$ ) or hypertriglyceridemia medication (C10AB); low HDL cholesterol ( $<1.03 / 1.30 \mathrm{mmol} / \mathrm{l}$ (men/women)) or medication (C10AB); hypertension (blood pressure $\geqslant 130 /$ $85 \mathrm{mmHg}$ ) or antihypertensive medication (C02, C03, C07, C08, C09); hyperglycemia (fasting glucose level $\geqslant 5.6 \mathrm{mmol} /$ 1) or antidiabetic medication (A10). Continuous metabolic measures incorporated medication use as described before (Vogelzangs et al, 2011; van Reedt Dortland et al, 2012). Triglyceride and glucose levels were ln-transformed to normalize distributions.

Overall dysregulation was quantified as the number of dichotomous inflammatory and metabolic dysregulations (range 0-8).

\section{Course of Depressive Disorders}

At the 2-year assessment, the CIDI was again administered to determine the presence of depressive disorders. A 6-month diagnosis at the 2-year follow-up indicates the presence of depression 1.5-2 years after baseline and was considered to indicate chronicity of a depressive disorder. This measure could include recurrent as well as persistent disorders. However, as both indicate a chronic character of the depression, for reasons of readability, we use the terms chronic and chronicity throughout this paper. An alternative measure of depression chronicity was based on the LCI (Lyketsos et al, 1994) administered at the 2-year followup, which assessed the presence of depressive symptoms during the previous 2 years for each month separately. Remission was defined as the first occurrence of reporting no depressive symptoms for three consecutive months. Recurrence was defined as the reoccurrence of symptoms with at least mild severity for at least 1 month under the condition that CIDI interview also confirmed the presence of a depressive disorder during follow-up. Persons were grouped in those with early remission (within 6 months; reference), late remission (after 6 months), remission and recurrence, and persistent course (no remission).

\section{Antidepressant Medication Use}

Next to type of antidepressant (SSRI, SNRI, TCA, TeCA), dosage and duration of use were assessed. Dosage was expressed as the daily dose used relative to the defined daily dose for each specific antidepressant according to ATC guidelines (WHO Collaborating Centre for Drug Statistics Methodology, 2013). Duration of antidepressant use was expressed in months before baseline. Antidepressant use was again assessed after 2 years, to determine whether persons stopped using antidepressants, switched to a different antidepressant, or continued using the same antidepressant.

\section{Covariates}

Sociodemographic characteristics included sex, age, and years of education. Several lifestyle characteristics were assessed as these can be associated with inflammatory and metabolic dysregulation, as well as with depression. Lifestyle factors included smoking status (never, former, current), alcohol intake ( $<1,1-14 / 1-21$ (women/men), $>14 / 21$ (women/men) drinks per week), and physical activity (measured with the International Physical Activity Questionnaire; Craig et al, 2003) in MET-minutes (ratio of energy expenditure during activity compared with rest times the number of minutes performing the activity) per week). Furthermore, number of self-reported chronic diseases for which persons received treatment (including cardiovascular disease, diabetes, lung disease, osteoarthritis or rheumatic disease, cancer, ulcer, intestinal problems, liver disease, epilepsy, and thyroid gland disease) and use of systemic anti-inflammatory medication (M01A, M01B, A07EB, A07EC) were assessed.

\section{Statistical Analyses}

All analyses were conducted using SPSS 20.0 (SPSS, Chicago, IL). Baseline characteristics were compared between antidepressant users who remitted and those who still/again had a depressive disorder after 2 years using $\chi^{2}$ Statistics for dichotomous and categorical variables and independent samples $t$-tests for continuous variables. In case of non-normal distribution, Mann-Whitney $U$-test was used.

Associations between baseline inflammatory and metabolic factors as predictors with the 2-year chronicity of depressive disorders as the outcome were assessed by means of logistic regression analyses. Separate analyses were performed for each dysregulation. First, analyses were adjusted for sociodemographics (ie, age, sex, education). Next, analyses were additionally adjusted for lifestyle and disease factors (ie, smoking status, alcohol intake, physical activity, number of chronic diseases; analyses with inflammatory markers were additionally adjusted for antiinflammatory medication). As several previous studies have found sex differences in the association between inflammatory and metabolic factors with depression (Howren et al, 2009; Kinder et al, 2004; Viinamaki et al, 2009; Vogelzangs et al, 2012), sex interactions were tested by including inflammatory/metabolic factor $\times$ sex interaction terms. In addition, to examine whether associations between inflammatory and metabolic dysregulations and depression chronicity were dependent on type or dosage of antidepressant use, interactions with SSRI vs SNRI/TCA/TeCA use and with defined daily dose $(<1,1,>1)$ were tested. When using depressive symptom course trajectories as the outcome, multinomial logistic regression analysis was used.

Because our study sample was not drug naive at baseline, inflammatory and metabolic factors could already have been influenced by antidepressant use, with varying influence according to duration of antidepressant use. To reduce heterogeneity in possible confounding effects of antidepressants on inflammatory and metabolic factors before baseline and to be able to examine non-response to antidepressants more strictly, a subgroup of persons was selected who only recently started antidepressant medication (ie, at most 3 months; $N=103$ ). To maintain sufficient sample size, a cutoff of 3 months was chosen and sensitivity analyses were performed using 2 and 4 months as cutoff. Within this group, the percentage of persons with chronic 
depression and with different course trajectories was compared according to the number of dysregulations using cross-tabs and $\chi^{2}$ tests for linear-by-linear association. Logistic regression analyses assessed fully adjusted associations between baseline inflammatory and metabolic factors with 2-year chronicity of depression.

\section{RESULTS}

Mean age of the study sample at baseline was 42.8 years $(\mathrm{SD}=11.3)$ and $63.8 \%$ were women. After 2 years, a depressive disorder was still/again present in $56.2 \%$. Antidepressant users with a remitted disorder $(N=138)$ vs those with a chronic disorder $(N=177)$ after 2 years did not differ significantly in sociodemographics, lifestyle, and most disease factors, nor in type, dosage, and duration of antidepressant use (Table 1). However, persons with a chronic course of depression did more often have diabetes at baseline and did more often switch to a different antidepressant during follow-up. Higher baseline levels of IL-6, triglycerides and glucose, a larger waist circumference, lower levels of HDL cholesterol as well as a higher number of inflammatory and metabolic dysregulations were (more often) found in persons with a chronic disorder compared with persons with remitted disorders.

Table 2 describes the adjusted associations between baseline inflammatory and metabolic dysregulation and the 2-year chronicity of depressive disorders in antidepressant users. As results were very similar after adjustment for sociodemographics and after additional adjustment for lifestyle and disease factors, only fully adjusted results are presented. Again, elevated IL-6, hypertriglyceridemia, low HDL cholesterol, and hyperglycemia significantly predicted depression chronicity. No significant associations were found for CRP, TNF- $\alpha$, abdominal obesity, and high blood pressure. Increasing the cutoff for high CRP to more clinically meaningful values (ie, $\mathrm{CRP} \geqslant 3$ and $\mathrm{CRP} \geqslant 5$ ) did not result in significant associations. Moreover, the total number of inflammatory and metabolic dysregulations significantly predicted depression chronicity. Persons showing $\geqslant 4$ dysregulations had $90 \%$ higher odds of still/again having a depressive disorder after 2 years as compared with those with 0-3 dysregulations. Examining different cutoff points yielded similar findings, with stronger associations found for higher cutoffs (ie, $\geqslant 3$ dysregulations: $O R=1.26$, $95 \% \mathrm{CI}=0.76-2.08 ; \geqslant 5$ dysregulations: $\mathrm{OR}=2.12,95 \%$ $\mathrm{CI}=1.17-3.85 ; \quad \geqslant 6 \quad$ dysregulations: $\mathrm{OR}=2.87, \quad 95 \%$ $\mathrm{CI}=1.34-6.17)$. Sex interactions were tested for all fully adjusted associations presented in Table 2, but no consistent sex interactions were found. In addition, interactions of inflammatory/metabolic dysregulations with SSRI vs SNRI/ TCA/TeCA use and with defined daily dose $(<1,1$, or $>1)$ in predicting 2-year depression chronicity were tested, but not found (all $p$ interaction $>0.10$ ). In fact, the strength of the association of inflammatory and metabolic dysregulation with depression chronicity was rather similar among SSRI users $(N=218$; number of dysregulations: $\mathrm{OR}=1.14,95 \%$ $\mathrm{CI}=0.68-1.52 ; \quad \geqslant 4 \quad$ dysregulations: $\mathrm{OR}=2.20, \quad 95 \%$ $\mathrm{CI}=0.83-5.81)$ and non-SSRI users $(N=97$; number of dysregulations: $\mathrm{OR}=1.16,95 \% \mathrm{CI}=0.99-1.37$; $\geqslant 4$ dysregulations: $\mathrm{OR}=1.70,95 \% \mathrm{CI}=0.86-3.34)$.
Next, course trajectories of depressive symptoms over 2 years were examined as an alternative outcome measure using multinomial logistic regression analysis. Figure 1 illustrates the association between the number of inflammatory and metabolic dysregulations and depression course trajectories. Persons with $4-5$ or 6-8 dysregulations had increased odds of late remission, remission and recurrence, or a persistent course relative to early remission compared with persons with 0-3 dysregulations. To improve power and since odds ratios were rather similar across different course trajectories and for those with 4-5 compared with those with 6-8 dysregulations, all results were combined in one summary logistic regression analysis, showing that those with $\geqslant 4$ dysregulations had a 2.26 increased odds (95\% CI $=1.17-4.35, p=0.02)$ of having a poor depressive symptoms course (ie, either late remission, remission and recurrence, or persistent course).

Within the subgroup of persons who only recently (ie, at most 3 months; median: 2 months) started antidepressant medication $(N=103)$, the percentage of persons showing chronicity of depression increased from $48.4 \%$ in those with $0-3$ dysregulations $(n=64)$ to $65.2 \%$ in those with $4-5$ dysregulations $(n=23)$ and $87.5 \%$ in those with $6-8$ dysregulations $(n=16) \quad(p=0.004)$. Figure 2 shows the percentage of persons with different depressive symptom course trajectories for persons with $0-3$ and for those with $\geqslant 4$ inflammatory and metabolic dysregulations. Owing to small numbers, those with 4-8 dysregulations were analyzed together. A recurrent or persistent course was much more common in those with $\geqslant 4$ dysregulations $(p=0.003)$.

Adjusted associations between baseline inflammatory and metabolic dysregulation and the 2-year chronicity of depressive disorders in recently started antidepressant users are presented in Table 2. Again, in particular IL-6, triglycerides, HDL cholesterol, and glucose were associated with depression chronicity with considerably larger ORs compared with those found in the whole group of antidepressant users. Every other inflammatory or metabolic dysregulation added an extra $59 \%$ odds for a chronic depression course. Persons showing $\geqslant 4$ dysregulations had a 6.85 increased odds of still/again having a depressive disorder after 2 years. Selecting persons with at most 2 or 4 months of antidepressant use, respectively, yielded similar results (ie, 2 months: $N=76$; number of dysregulations: $\mathrm{OR}=1.83, \quad 95 \% \quad \mathrm{CI}=1.24-2.70 ; \quad \geqslant 4$ dysregulations: $\mathrm{OR}=13.56, \quad 95 \% \quad \mathrm{CI}=2.44-75.50 ; 4$ months: $N=120$; number of dysregulations: $\mathrm{OR}=1.45,95 \% \mathrm{CI}=1.11-1.89$; $\geqslant 4$ dysregulations: $\mathrm{OR}=5.06,95 \% \mathrm{CI}=1.75-14.68)$.

\section{DISCUSSION}

In this study, we prospectively examined whether inflammatory and metabolic dysregulation predicted the 2-year course of depressive disorders among antidepressant users. The results show that elevated IL-6, low HDL cholesterol, hypertriglyceridemia, and hyperglycemia were associated with chronicity of depressive disorders. A greater number of inflammatory and metabolic dysregulations was associated with an increased risk of chronic depression. These findings were especially true within persons who only recently started antidepressant medication, which could possibly 
Inflammatory and metabolic factors and depression course

N Vogelzangs et al

Table I Baseline Characteristics of Antidepressant Users With and Without a Depressive Disorder After 2 Years

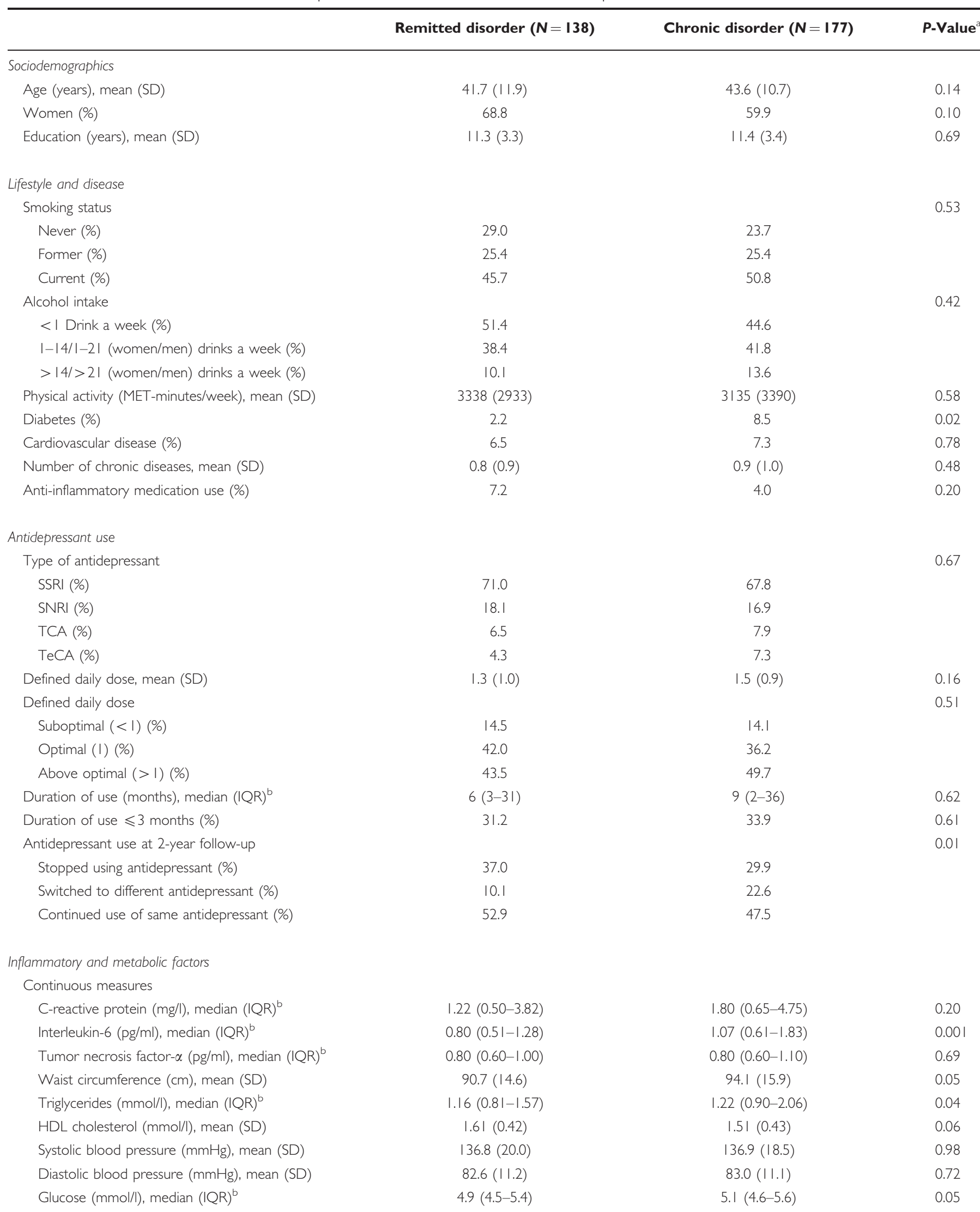




\begin{tabular}{|c|c|c|c|}
\hline & Remitted disorder $(N=138)$ & Chronic disorder $(N=177)$ & $P$-Value ${ }^{a}$ \\
\hline \multicolumn{4}{|l|}{ Dichotomous measures } \\
\hline High C-reactive proteinc (\%) & 46.7 & 52.6 & 0.30 \\
\hline High interleukin-6 ${ }^{c}(\%)$ & 40.0 & 57.7 & 0.002 \\
\hline High tumor necrosis factor- $\alpha^{c}(\%)$ & 52.6 & 54.0 & 0.80 \\
\hline Abdominal obesity (\%) & 42.3 & 40.7 & 0.77 \\
\hline Hypertension (\%) & 64.5 & 63.6 & 0.88 \\
\hline Hyperglycemia (\%) & 12.1 & 25.6 & 0.003 \\
\hline \multicolumn{4}{|l|}{ Summary measures } \\
\hline Total number of dysregulations, mean (SD) & $2.9(1.7)$ & $3.5(2.1)$ & 0.005 \\
\hline
\end{tabular}

Abbreviations: $\mathrm{HDL}=$ high-density lipoprotein; SNRI = serotonin-norepinephrine reuptake inhibitor; SSRI = selective serotonin reuptake inhibitor; $\mathrm{TCA}=$ tricyclic antidepressant; $\mathrm{TeCA}=$ tetracyclic antidepressant.

${ }^{a}$ On the basis of $\chi^{2}$ test for dichotomous and categorical variables and independent samples $t$-test for continuous variables.

bMann-Whitney U-test was used because of non-normal distribution.

${ }^{c}$ High is defined as above median (C-reactive protein $\geqslant 1.58$; interleukin- $6 \geqslant 0.95$; tumor necrosis factor- $\alpha \geqslant 0.80$ ).

indicate that such dysregulation reflects poor response to antidepressants.

The results of our comparatively large study are in line with the few previous small studies showing that high inflammation, indicated by either increased blood levels, gene expression, or genetic polymorphisms, prospectively predicted non-response after the start of conventional antidepressant treatment (Eller et al, 2008; Lanquillon et al, 2000; Cattaneo et al, 2013; Yu et al, 2003; Baune et al, 2010). Although our subjects were not drug naive at the start of the study, strongest associations were found among those recently started on antidepressants, such that long-term effects of antidepressant use on inflammatory or metabolic dysregulation could not have influenced our results. On the other hand, the production of cytokines such as IL-6 may increase owing to the acute administration of antidepressant medication (Kubera et al, 2000). Therefore, it is possible that it is not the inflammatory or metabolic dysregulation before treatment, but rather a large acute inflammatory or metabolic response to starting antidepressant use, which predicts non-response. Furthermore, among those with chronic depression, more persons switched to a different antidepressant, likely reflecting insufficient response to the first antidepressant. This is further evidence that depression chronicity among antidepressant users is an indication of reduced treatment response. Our results elaborate on previous findings by showing chronicity of depression even after a period of 2 years. Furthermore, our findings demonstrated that not only inflammatory dysregulation, but in addition metabolic dysregulation, such as dyslipidemia and hyperglycemia, predicts a poor depression course among antidepressant users, suggesting that metabolic dysregulation may also indicate poor treatment response to antidepressants.

Poor treatment response to conventional antidepressants in a subgroup of patients may be further evidence of the existence of an etiologically different subtype of depression with inflammatory and metabolic dysregulation, which is clinically relevant and which we have previously labeled 'metabolic depression' (Vogelzangs et al, 2011). Additional evidence for a specific inflammatory/metabolic subtype comes from recent findings of the NESDA study showing that these dysregulations are restricted to patients with an atypical symptom profile, and are not found among those with melancholic features (Lamers et al, 2013). Alternatively, poor treatment response could be the result of inflammatory and metabolic dysregulation having direct negative effects on the monoamine system, such as increasing the activity of monoamine transporters ( $\mathrm{hhu}$ et al, 2006) and reducing the concentration of monoamine precursors (Widner et al, 2002) and monoamine biosynthesis (Neurauter et al, 2008), which counterbalance the effects of antidepressant medication. This may be true for all types of conventional antidepressants as we found that inflammatory and metabolic dysregulation predicted chronicity of depression similarly across SSRI, SNRI, and TCA/TeCA users.

We and others have found that inflammatory and metabolic dysregulation is more prominent in persons using antidepressants, in particular in those using SNRI, TCA, or TeCA (Hamer et al, 2011; Pyykkonen et al, 2012; van Reedt Dortland et al, 2010; Vogelzangs et al, 2012), although more beneficial inflammatory profiles have been found in persons using SSRI (Miller et al, 2009; Vogelzangs et al, 2012). Furthermore, two recent meta-analyses showed that SSRI treatment was associated with reductions in inflammatory levels (ie, CRP, IL-6, IL-1 $\beta$, and nonsignificant trends for TNF- $\alpha$ and IL-10), while this was not found for other types of antidepressants (Hannestad et al, 2011; Hiles et al, 2012). One small study did find that IL-6 and TNF- $\alpha$ levels decreased after treatment with amitriptyline, but these decreases were only found in treatment responders, while IL-6 levels even increased in treatment nonresponders (Lanquillon et al, 2000). This could imply that 
Table 2 Associations ${ }^{\mathrm{a}}$ Between Baseline Inflammatory and Metabolic Dysregulation and 2-Year Depressive Disorder Chronicity in Antidepressant Users

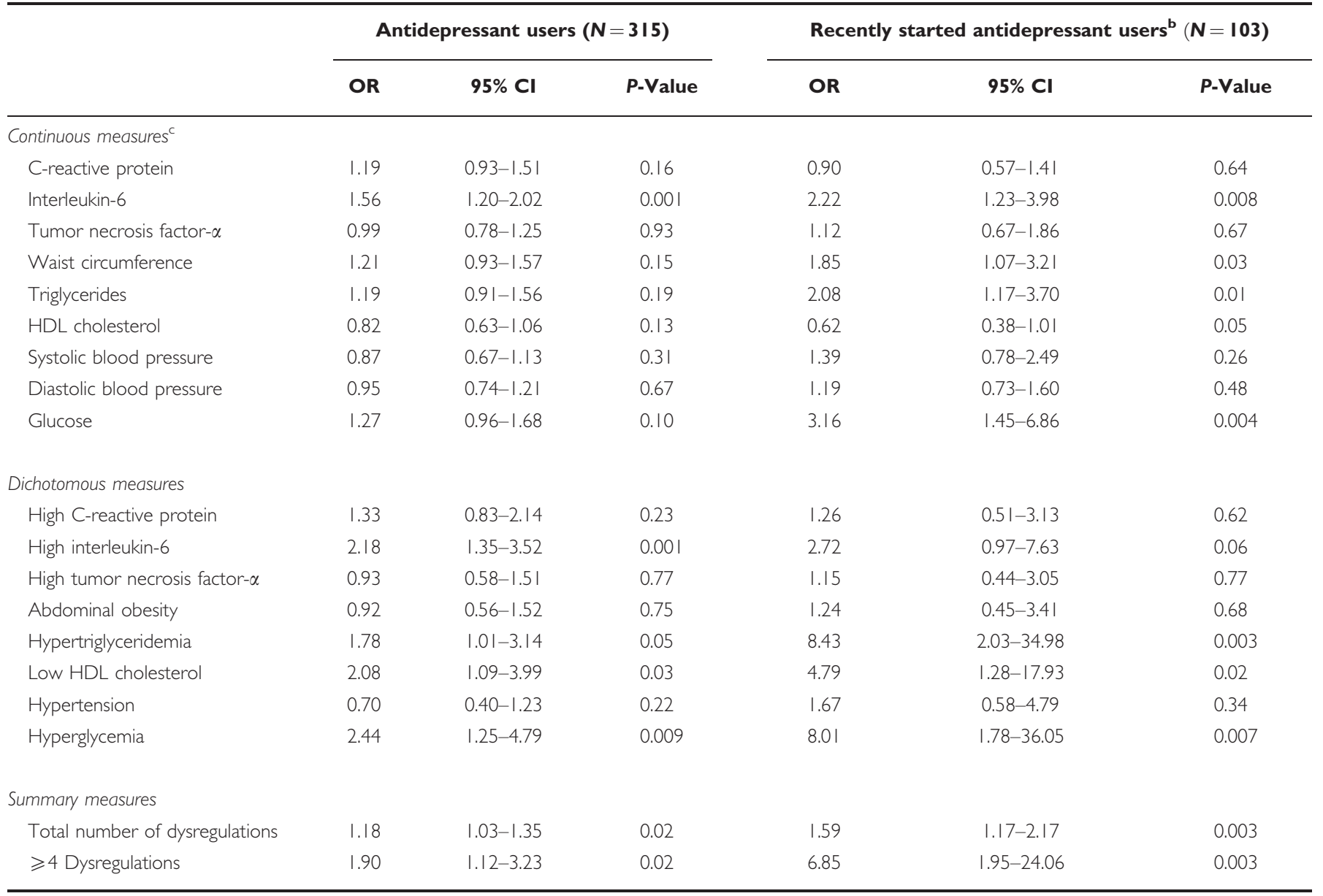

Abbreviation: $\mathrm{HDL}=$ high-density lipoprotein.

${ }^{a}$ On the basis of logistic regression analyses adjusted for age, sex, education, smoking status, alcohol intake, physical activity, and number of chronic diseases; inflammatory markers were additionally adjusted for anti-inflammatory medication.

bo-3 Months of antidepressant use.

${ }^{c}$ Per SD of normally distributed variable $-\ln (C R P): S D=1.30 ; \ln (\mathrm{IL}-6)$ : $S D=0.96 ; \ln (T N F-\alpha)$ : $S D=0.61$; waist circumference: $S D=\mid 5.4 ; \ln ($ triglycerides): $\mathrm{SD}=0.58$; HDL cholesterol: $\mathrm{SD}=0.43$; systolic blood pressure: $\mathrm{SD}=19.2$; diastolic blood pressure: $\mathrm{SD}=1 \mathrm{I} .1$; $\ln$ (glucose): $\mathrm{SD}=0.18$. No corrections for multiple testing were applied.

among depressed persons with inflammatory and metabolic dysregulation, traditional antidepressants may not only be less effective but non-SSRI agents may also even worsen the inflammatory and metabolic profile of these patients.

What do these study findings imply for depression treatment? Depression is a heterogeneous disorder and currently available treatments, including the prevention of relapse, is only effective in about a third to half of our patients (Pigott et al, 2010). Identifying depression subtypes can help targeting new interventions. If a specific subgroup of depressed persons with inflammatory and metabolic dysregulation exists, these persons may benefit from alternative treatments, such as treatments targeting the immune or metabolic system. Preliminary evidence from studies among patients treated with anti-inflammatory agents for other indications suggests that these agents may have beneficial effects on mood (Muller et al, 2004). One study found positive effects on mood in medically healthy, major depressed patients (Muller et al, 2006).
Moreover, a recent trial among persons with treatmentresistant depression showed that an add-on treatment with the TNF- $\alpha$ antagonist infliximab improved depressive symptoms only among those who showed increased inflammation before treatment (Raison et al, 2013). What is even more is that, in this same trial, response to infliximab could be predicted by gene expression reflecting alterations in lipid and glucose metabolism (Mehta et al, 2013). Furthermore, behavioral interventions such as exercise have shown to normalize immune and metabolic dysregulation (You and Nicklas, 2008), as well as to improve depressive symptoms to some degree (Mead et al, 2009), and may therefore be an indicated treatment for this subgroup of depressed persons with inflammatory and metabolic dysregulation. This idea is supported by a recent study showing that exercise treatment appeared to be more effective in reducing depressive symptoms among those persons with high baseline levels of TNF- $\alpha$ (Rethorst $e t$ al, 2013). However, at this moment, these considerations for 


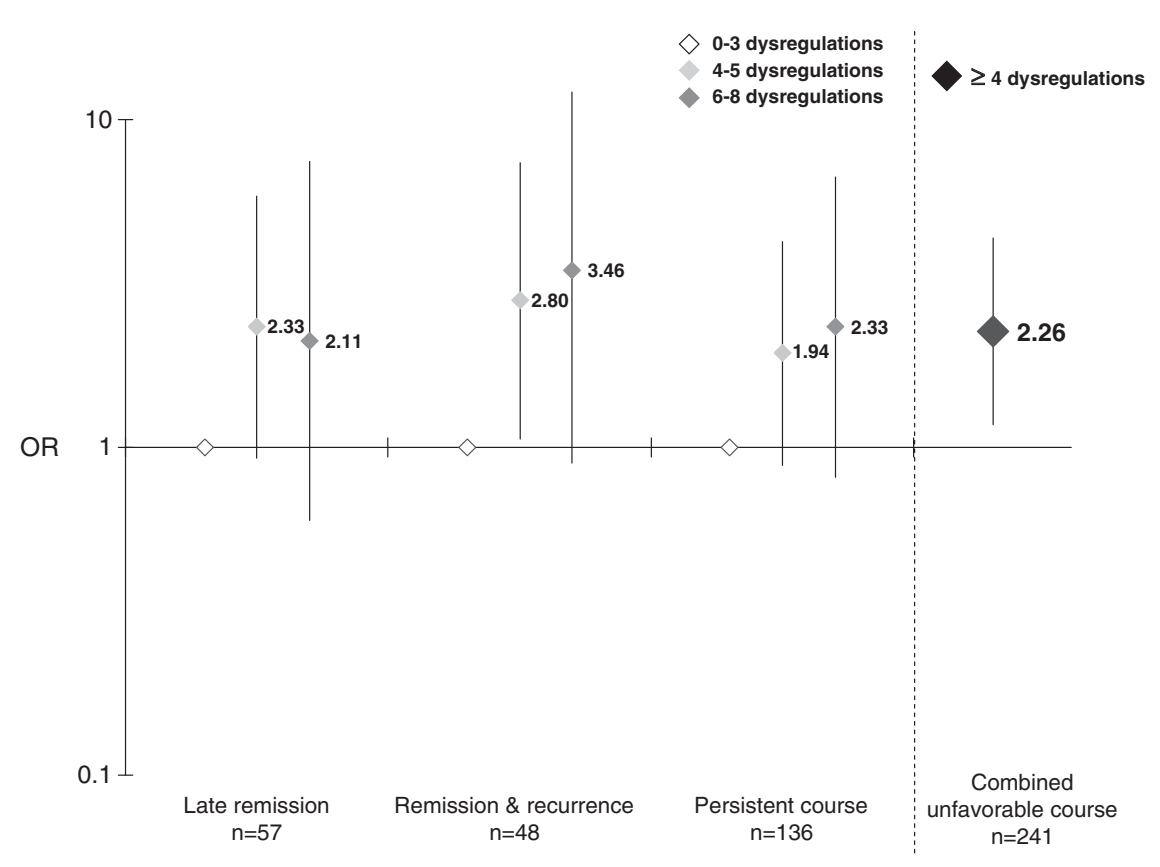

Figure I Adjusted odds ratio (OR) for 2-year depressive symptoms course trajectories in antidepressant users according to the number of inflammatory and metabolic dysregulations. On the basis of (multinomial) logistic regression analyses, with early remission as the reference group ( $N=73$ ) adjusted for age, sex, education, smoking status, alcohol intake, physical activity, number of chronic diseases, and anti-inflammatory medication; the vertical dashed line separates two different analyses; combined unfavorable course indicates either late remission, remission and recurrence, or persistent course.

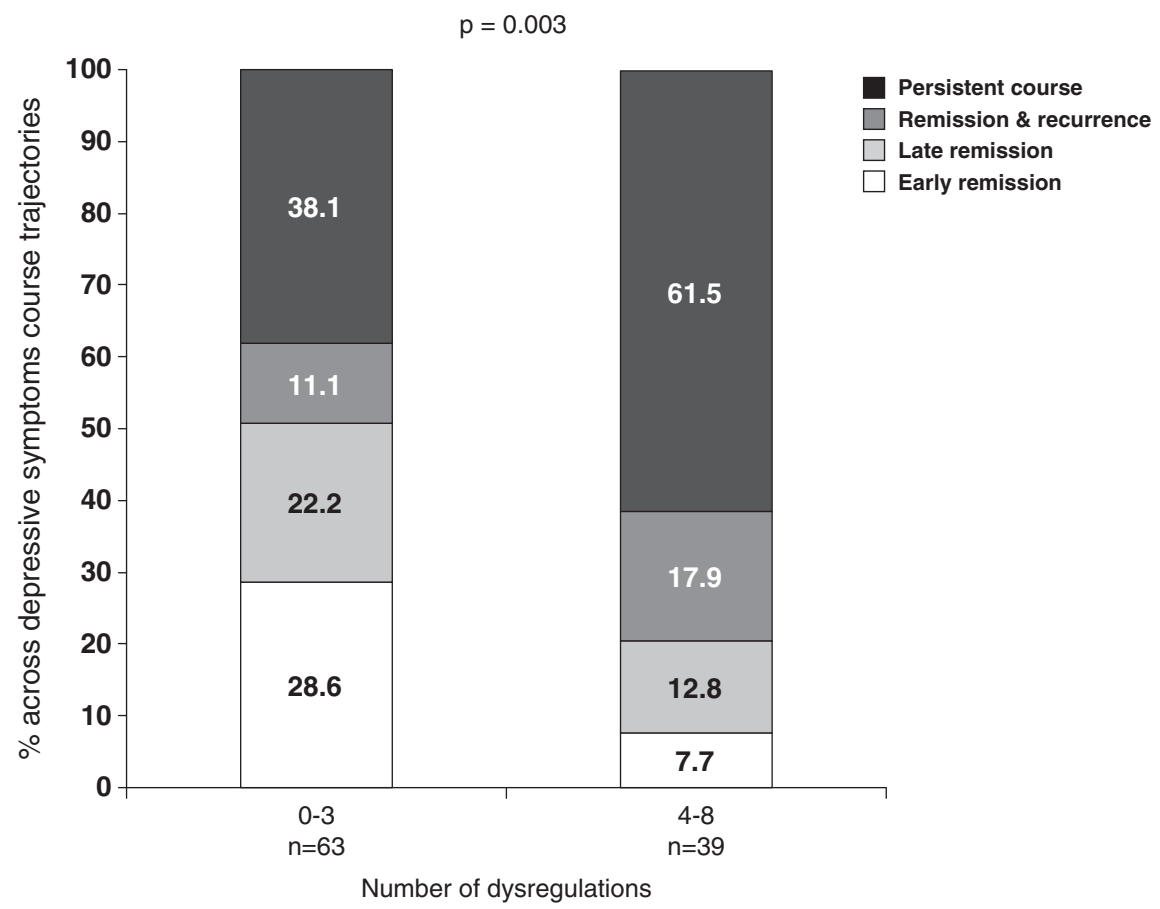

Figure 2 Course trajectories for recently started antidepressant users according to the number of inflammatory and metabolic dysregulations. On the basis of cross-tab and $\chi^{2}$ statistic for linear-by-linear association.

treatment implications are still speculative and should be confirmed in longitudinal and experimental studies.

Of the different inflammatory markers assessed, strong associations were found for IL-6, while no associations with depression chronicity were found for CRP and TNF- $\alpha$. This is only partly in line with our previous cross-sectional report in this same sample in which depression status was most strongly associated with CRP, followed by IL-6 and not with TNF- $\alpha$ (Vogelzangs et al, 2012). Also, meta-analyses have implicated a role for CRP and TNF- $\alpha$ in depression next to IL-6 (Dowlati et al, 2010; Howren et al, 2009), and TNF- $\alpha$ has been found to be predictive of non-response to 
escitalopram (Eller et al, 2008). Possibly, elevated CRP reflects a depressive state, but lacks specificity to predict course or treatment response. The fact that CRP is released in response to IL- 6 and that IL-6, but not CRP, can cross the blood-brain barrier (Banks et al, 1994) may also suggest a more prominent prognostic role for IL-6 compared with CRP. As for TNF- $\alpha$, intra- and interassay coefficients of variation were, at least in our sample, somewhat higher for TNF- $\alpha$ compared with CRP and IL-6, which may partly explain the weaker associations for TNF- $\alpha$ in our sample. Future research has to elucidate whether specifically IL-6 is associated with depression chronicity or whether other inflammation markers are of additional relevance.

With regard to the metabolic factors, associations with depression chronicity were found for lipid dysregulations and hyperglycemia, which have also been associated with the onset of depression (Pan et al, 2012; Viinamaki et al, 2009). We found no clear associations between abdominal obesity and hypertension with a chronic course of depression. Previous research on metabolic syndrome and depression has indeed found that hypertension is the component contributing least to this association (Pan et al, 2012). Abdominal obesity, however, has consistently been associated with depression and depression onset (Xu et al, 2011; Vogelzangs et al, 2010, 2011). Possibly, once depression is present, abdominal obesity is of less importance for the persistence of depression. We previously found in an older sample that waist circumference did predict onset of depressive symptoms, but was not significantly related to the persistence of depression (Vogelzangs et al, 2011). Possibly the consequences of abdominal obesity (ie, inflammation, lipid dysregulations) become more important in hindering recovery of depressive symptoms than abdominal obesity itself.

Important strengths of our study are that it is one of the first and largest to examine the prospective association between several inflammatory as well as metabolic dysregulations with the course of depressive disorders assessed according to DSM-IV diagnoses. Furthermore, we were able to test for sex interactions and to adjust for a large set of possible confounders. However, some limitations need to be acknowledged. Inflammatory and metabolic factors were only measured at baseline. Perhaps, even more clear associations could be found in persons showing continuing inflammatory or metabolic dysregulation over the 2-year follow-up. In addition, as this is an observational study, we cannot disentangle natural unfavorable course from treatment resistance. Experimental research in initially antidepressantnaive patients has to establish ultimately whether depressed persons with inflammatory and metabolic dysregulation are at risk of non-response to traditional antidepressants.

In conclusion, our study shows that in antidepressant users, a chronic, poor course of depressive disorders is predicted by elevated IL-6 levels, hypertriglyceridemia, low HDL cholesterol, and hyperglycemia. This may suggest a possible poor response to traditional antidepressants in these persons. Future longitudinal and experimental studies need to confirm our findings and investigate whether alternative treatments (eg, anti-inflammatory medication, exercise) are better able to improve depressive disorder course in persons with inflammatory and metabolic dysregulation.

\section{FUNDING AND DISCLOSURE}

The infrastructure for the NESDA study (http://www. nesda.nl) is funded through the Geestkracht program of the Netherlands Organization for Health Research and Development (Zon-Mw, Grant No. 10-000-1002) and is supported by participating universities and mental healthcare organizations (VU University Medical Center, GGZ inGeest, Arkin, Leiden University Medical Center, GGZ Rivierduinen, University Medical Center Groningen, Lentis, GGZ Friesland, GGZ Drenthe, Institute for Quality of Health Care (IQ Healthcare), Netherlands Institute for Health Services Research (NIVEL), and Netherlands Institute of Mental Health and Addiction (Trimbos). NV was supported through a fellowship from the EMGO Institute for Health and Care Research, BP through a VICI grant (NWO Grant No. 91811602) and PdJ through a VICI grant (NWO Grant No. 91812607). Assaying of inflammatory markers was supported by the Neuroscience Campus Amsterdam. ATFB received grants for research from Eli Lilly, Astra Zenica, Janssen, and Shire and as a speaker from Lundbeck and Eli Lilly. All other authors declare no conflict of interest.

\section{REFERENCES}

American Psychiatric Association (2001). Diagnostic and Statistical Manual of Mental Disorders 4th ednAmerican Psychiatric Association: Washington, DC.

Banks WA, Kastin AJ, Gutierrez EG (1994). Penetration of interleukin-6 across the murine blood-brain barrier. Neurosci Lett 179: 53-56.

Baune BT, Dannlowski U, Domschke K, Janssen DG, Jordan MA, Ohrmann P et al (2010). The interleukin 1 beta (IL1B) gene is associated with failure to achieve remission and impaired emotion processing in major depression. Biol Psychiatry 67: 543-549.

Buist-Bouwman MA, de GR, Vollebergh WA, Alonso J, Bruffaerts R, Ormel J (2006). Functional disability of mental disorders and comparison with physical disorders: a study among the general population of six European countries. Acta Psychiatr Scand 113: 492-500.

Cattaneo A, Gennarelli M, Uher R, Breen G, Farmer A, Aitchison $\mathrm{KJ}$ et al (2013). Candidate genes expression profile associated with antidepressants response in the GENDEP study: differentiating between baseline 'predictors' and longitudinal 'targets'. Neuropsychopharmacology 38: 377-385.

Craig CL, Marshall AL, Sjostrom M, Bauman AE, Booth ML, Ainsworth BE et al (2003). International physical activity questionnaire: 12 -country reliability and validity. Med Sci Sports Exerc 35: 1381-1395.

Dowlati Y, Herrmann N, Swardfager W, Liu H, Sham L, Reim EK et al (2010). A meta-analysis of cytokines in major depression. Biol Psychiatry 67: 446-457.

Duivis HE, de JP, Penninx BW, Na BY, Cohen BE, Whooley MA (2011). Depressive symptoms, health behaviors, and subsequent inflammation in patients with coronary heart disease: prospective findings from the heart and soul study. Am J Psychiatry 168: 913-920.

Eller T, Vasar V, Shlik J, Maron E (2008). Pro-inflammatory cytokines and treatment response to escitalopram in major depressive disorder. Prog Neuropsychopharmacol Biol Psychiatry 32: 445-450.

Grundy SM, Cleeman JI, Daniels SR, Donato KA, Eckel RH, Franklin BA et al (2005). Diagnosis and management of the metabolic syndrome: an American Heart Association/National 
Heart, Lung, and Blood Institute Scientific Statement. Circulation 112: 2735-2752.

Hamer M, Batty GD, Marmot MG, Singh-Manoux A, Kivimaki M (2011). Anti-depressant medication use and C-reactive protein: results from two population-based studies. Brain Behav Immun 25: $168-173$.

Hannestad J, DellaGioia N, Bloch M (2011). The effect of antidepressant medication treatment on serum levels of inflammatory cytokines: a meta-analysis. Neuropsychopharmacology 36: 2452-2459.

Hiles SA, Baker AL, de MT, Attia J (2012). Interleukin-6, C-reactive protein and interleukin-10 after antidepressant treatment in people with depression: a meta-analysis. Psychol Med 42: 2015-2026.

Howren MB, Lamkin DM, Suls J (2009). Associations of depression with C-reactive protein, IL-1, and IL-6: a meta-analysis. Psychosom Med 71: 171-186.

Kessler RC, Avenevoli S, Costello EJ, Georgiades K, Green JG, Gruber MJ et al (2012). Prevalence, persistence, and sociodemographic correlates of DSM-IV disorders in the National Comorbidity Survey Replication Adolescent Supplement. Arch Gen Psychiatry 69: 372-380.

Kinder LS, Carnethon MR, Palaniappan LP, King AC, Fortmann SP (2004). Depression and the metabolic syndrome in young adults: findings from the Third National Health and Nutrition Examination Survey. Psychosom Med 66: 316-322.

Kubera M, Simbirtsev A, Mathison R, Maes M (2000). Effects of repeated fluoxetine and citalopram administration on cytokine release in C57BL/6 mice. Psychiatry Res 96: 255-266.

Lamers F, Hoogendoorn AW, Smit JH, Van DR, Zitman FG, Nolen WA et al (2012). Sociodemographic and psychiatric determinants of attrition in the Netherlands Study of Depression and Anxiety (NESDA). Compr Psychiatry 53: 63-70.

Lamers F, Vogelzangs N, Merikangas KR, de JP, Beekman AT, Penninx BW (2013). Evidence for a differential role of HPA-axis function, inflammation and metabolic syndrome in melancholic versus atypical depression. Mol Psychiatry 18: 692-699.

Lanquillon S, Krieg JC, ing-Abu-Shach U, Vedder H (2000). Cytokine production and treatment response in major depressive disorder. Neuropsychopharmacology 22: 370-379.

Lyketsos CG, Nestadt G, Cwi J, Heithoff K, Eaton WW (1994). The life chart interview: a standardized method to describe the course of psychopathology. Int J Methods Psychiatric Res 4: 143-155.

Mathers CD, Loncar D (2006). Projections of global mortality and burden of disease from 2002 to 2030. PLoS Med 3: e442.

Mead GE, Morley W, Campbell P, Greig CA, McMurdo M, Lawlor DA (2009). Exercise for depression. Cochrane Database Syst Rev CD004366.

Mehta D, Raison CL, Woolwine BJ, Haroon E, Binder EB, Miller $\mathrm{AH}$ et al (2013). Transcriptional signatures related to glucose and lipid metabolism predict treatment response to the tumor necrosis factor antagonist infliximab in patients with treatmentresistant depression. Brain Behav Immun 31: 205-215.

Miller AH, Maletic V, Raison CL (2009). Inflammation and its discontents: the role of cytokines in the pathophysiology of major depression. Biol Psychiatry 65: 732-741.

Muller N, Riedel M, Schwarz MJ (2004). Psychotropic effects of COX-2 inhibitors-a possible new approach for the treatment of psychiatric disorders. Pharmacopsychiatry 37: 266-269.

Muller N, Schwarz MJ, Dehning S, Douhe A, Cerovecki A, Goldstein-Muller B et al (2006). The cyclooxygenase-2 inhibitor celecoxib has therapeutic effects in major depression: results of a double-blind, randomized, placebo controlled, add-on pilot study to reboxetine. Mol Psychiatry 11: 680-684.

Neurauter G, Schrocksnadel K, Scholl-Burgi S, Sperner-Unterweger B, Schubert C, Ledochowski M et al (2008). Chronic immune stimulation correlates with reduced phenylalanine turnover. Curr Drug Metab 9: 622-627.
Pan A, Keum N, Okereke OI, Sun Q, Kivimaki M, Rubin RR et al (2012). Bidirectional association between depression and metabolic syndrome: a systematic review and meta-analysis of epidemiological studies. Diabetes Care 35: 1171-1180.

Penninx BW, Beekman AT, Smit JH, Zitman FG, Nolen WA, Spinhoven P et al (2008). The Netherlands Study of Depression and Anxiety (NESDA): rationale, objectives and methods. Int $J$ Methods Psychiatr Res 17: 121-140.

Penninx BW, Nolen WA, Lamers F, Zitman FG, Smit JH, Spinhoven P et al (2011). Two-year course of depressive and anxiety disorders: results from the Netherlands Study of Depression and Anxiety (NESDA). J Affect Disord 133: 76-85.

Pigott HE, Leventhal AM, Alter GS, Boren JJ (2010). Efficacy and effectiveness of antidepressants: current status of research. Psychother Psychosom 79: 267-279.

Pyykkonen AJ, Raikkonen K, Tuomi T, Eriksson JG, Groop L, Isomaa B (2012). Association between depressive symptoms and metabolic syndrome is not explained by antidepressant medication: results from the PPP-Botnia Study. Ann Med 44: 279-288.

Raison CL, Rutherford RE, Woolwine BJ, Shuo C, Schettler P, Drake DF et al (2013). A randomized controlled trial of the tumor necrosis factor antagonist infliximab for treatmentresistant depression: the role of baseline inflammatory biomarkers. JAMA Psychiatry 70: 31-41.

Rethorst CD, Toups MS, Greer TL, Nakonezny PA, Carmody TJ, Grannemann BD et al (2013). Pro-inflammatory cytokines as predictors of antidepressant effects of exercise in major depressive disorder. Mol Psychiatry 18: 1119-1124.

Rhebergen D, Beekman AT, Graaf R, Nolen WA, Spijker J, Hoogendijk WJ et al (2009). The three-year naturalistic course of major depressive disorder, dysthymic disorder and double depression. J Affect Disord 115: 450-459.

Spijker J, de GR, Bijl RV, Beekman AT, Ormel J, Nolen WA (2002). Duration of major depressive episodes in the general population: results from The Netherlands Mental Health Survey and Incidence Study (NEMESIS). Br J Psychiatry 181: 208-213.

Sutherland JP, McKinley B, Eckel RH (2004). The metabolic syndrome and inflammation. Metab Syndr Relat Disord 2: 82-104.

van Reedt Dortland AK, Giltay EJ, van VT, Zitman FG, Penninx BW (2010). Metabolic syndrome abnormalities are associated with severity of anxiety and depression and with tricyclic antidepressant use. Acta Psychiatr Scand 122: 30-39.

van Reedt Dortland AK, Giltay EJ, van VT, Zitman FG, Penninx BW (2012). Personality traits and childhood trauma as correlates of metabolic risk factors: the Netherlands Study of Depression and Anxiety (NESDA). Prog Neuropsychopharmacol Biol Psychiatry 36: 85-91.

Viinamaki H, Heiskanen T, Lehto SM, Niskanen L, KoivumaaHonkanen H, Tolmunen T et al (2009). Association of depressive symptoms and metabolic syndrome in men. Acta Psychiatr Scand 120: 23-29.

Vogelzangs N, Beekman AT, Boelhouwer IG, Bandinelli S, Milaneschi Y, Ferrucci L et al (2011). Metabolic depression: a chronic depressive subtype? Findings from the InCHIANTI study of older persons. J Clin Psychiatry 72: 598-604.

Vogelzangs N, Duivis HE, Beekman AT, Kluft C, Neuteboom J, Hoogendijk W et al (2012). Association of depressive disorders, depression characteristics and antidepressant medication with inflammation. Transl Psychiatry 2: e79.

Vogelzangs N, Kritchevsky SB, Beekman AT, Brenes GA, Newman $A B$, Satterfield S et al (2010). Obesity and onset of significant depressive symptoms: results from a prospective communitybased cohort study of older men and women. J Clin Psychiatry 71: 391-399.

WHO Collaborating Centre for Drug Statistics MethodologyAvailable at http://www.whocc.no/(accessed 1 May 2013). 
Widner B, Laich A, Sperner-Unterweger B, Ledochowski M, Fuchs D (2002). Neopterin production, tryptophan degradation, and mental depression-what is the link? Brain Behav Immun 16: 590-595.

Wittchen HU (1994). Reliability and validity studies of the WHOComposite International Diagnostic Interview (CIDI): a critical review. J Psychiatr Res 28: 57-84.

Xu Q, Anderson D, Lurie-Beck J (2011). The relationship between abdominal obesity and depression in the general population: a systematic review and meta-analysis. Obes Res Clin Pract 5: e267-e278.
You T, Nicklas BJ (2008). Effects of exercise on adipokines and the metabolic syndrome. Curr Diab Rep 8: 7-11.

Yu YW, Chen TJ, Hong CJ, Chen HM, Tsai SJ (2003). Association study of the interleukin-1 beta (C-511T) genetic polymorphism with major depressive disorder, associated symptomatology, and antidepressant response. Neuropsychopharmacology 28: $1182-1185$.

Zhu CB, Blakely RD, Hewlett WA (2006). The proinflammatory cytokines interleukin-1beta and tumor necrosis factor-alpha activate serotonin transporters. Neuropsychopharmacology 31: 2121-2131. 